\title{
Selim tiroid hastalıklarının cerrahi tedavisinde subtotal ve total tiroidektominin erken ve geç dönem sonuçlarının karşılaştırılması
}

\author{
Kemal Arslan', Ersin Turan¹, Mehmet Ali Eryılmaz1, Emet Ebru Nazik1, Osman Doğru
}

ÖZET:

Selim tiroid hastalıklarının cerrahi tedavisinde subtotal ve total tiroidektominin erken ve geç dönem sonuçlarının karşılaştırılması

Amaç: Son yıllarda benign tiroid hastalıklarında total tiroidektomi (TT) uygulanmakta ve önerilmektedir. Ancak TT'nin komplikasyonlarının bilateral subtotal (BST)'den daha fazla olduğu bildirilmiş ve bu endişe halen devam etmektedir. Bu çalışmanın amacı selim tiroidektomi hastalıklarının tedavisinde TT ile BST ameliyatlarının erken ve geç dönem komplikasyonlarının karşılaştırılmasıdır.

Gereç ve Yöntem: Konya Eğitim ve Araştırma Hastanesi Genel Cerrahi Kliniğinde 2004-2008 yılları arasında yapılan BST ve TT ameliyatlarının erken ve geç dönem komplikasyonları incelendi.

Bulgular: BST grubunda 96, TT grubunda 110 hasta çalışmaya alındı. Takip süresi BST grubunda $69.4 \pm 8.3$ ay (60-84), TT grubunda $52.7 \pm 5.3$ ay (48-60) idi ve BST grubunda daha uzundu. En sık tespit edilen komplikasyon geçici hipokalsemi idi. Hipokalsemi BST grubunda 10 hastada (\%9.1), TT grubunda 7 hastada (\%7.3) saptandı. Geçici rekürren laringeal sinir paralizisi BST grubunda 5 hastada (\%5.2), TT grubunda 7 hastada (\%6.4) saptandı. BST grubunda hematom 4 (\%4.2), seroma 3 (\%3.1), flep ödemi 5 (\%5.2) hastada gelişti. TT grubunda hematom $1(\% 1.0)$, seroma 5 (\%4.5) ve flep ödemi 4 (\%4.5) hastada saptandı. Kalıcı hipokalsemi BST grubunda 2 hastada (\%2.1), TT grubunda 3 hastada (\%2.7) gelişti. BST grubunda 1, TT grubunda 2 hastada (\%1.5) kalııı rekürrenlaringeal paralizisi gelişti. Demografik özellikler ve komplikasyonlar açısından gruplar arasında anlamlı fark yoktu. BST grubunda $6(\% 6.3)$ hastada nüks görüldü.

Tartışma ve Sonuç: Cerrahi gerektiren selim tiroid hastalıklarında TT erken ve geç komplikasyonları arttırmamaktadır. BST sonrası nüks intimali vardır ve bu nedenle tekrar ameliyat gerektirebilmektedir. Nüks tiroid ameliyatlarından sonra primer ameliyatlara göre komplikasyonlar belirgin şekilde daha fazladır. Bu nedenle benigntiroid hastalıklarında eğer cerrahi gerekliyse TT uygulanmalıdır.

Anahtar kelimeler: Subtotal tiroidektomi, total tiroidektomi, rekürren laringeal paralizisi, hipokalsemi

\section{ABSTRACT:}

Comparison of early and late results of total and subtotal thyroidectomy for benign thyroid disease

Background: Recently, total thyroidectomy (TT) has been performed and suggested in benign thyroid disease. But it was reported that the complications of total thyroidectomy was more than bilateral subtotal thyroidectomy (BST) this is still a topic of debate. Purpose of the present study is comparison of complications of BST and TT operations in treatment of benign thyroid disease.

Materials and Methods: Early and late complications of TT and BST operations were retrospectively evaluated, which were performed in General Surgery Clinic of Konya Training and Research Hospital inbetween 2004-2008.

Results:The study involved 96 patients in BST group and 110 patients in TT group. Follow-up period was $69.4 \pm 8.3$ months in BST group, 52.7 \pm 5.3 months in TT group.Transient hypocalcemia were detected in 10 patients (9.1\%) in BST group and 7 patients (7.3\%) in TT group. Transient recurrent laryngeal nerve paralysis developed in 12 patients $(7.2 \%)$; 5 patients $(5.2 \%)$ in BST group and 7 patients $(6.4 \%)$ in TT group. Hematoma in 4 patients (4.2\%), seroma in 3 patients (3.1\%), flap edema in 5 patients (5.2\%) occured in BST group. In TT group, there had been; hematoma in 1 patients $(1.0 \%)$, seroma in 5 patients (4.5\%), flap edema in 4 patients $(4.5 \%)$.

Permanent hypocalcemia occured in 2 patients (2.1\%) in BST group and 3 patients (2.7\%) in TT group. Permanent recurrent laryngeal nerve paralysis developed in 1 patient (1.0\%) in BST group and 2 patients (1.5\%) in TT group. There was no statistically significant difference inbetween the groups about complications and demographic characteristics. Recurrence was seen in 6 patients (6.3\%) in BST group.

Discussion and Conclusion: TT not increase early and late complications in bengn thyroid diease requiring sugery. BSThas arisk of recurrenceaftersurgeryand thereforemust be carried outagain.Complications in secondary thyroidectomy in patients with prior thyroid surgery are very high.Therefore, if surgery is necessary in benign thyroid diseases should be TT.

Key words: Subtotal thyroidectomy, total thyroidectomy, paralysis of recurrent laryngeal nerve, hypocalcemia

Ş.E.E.A.H. Tıp Bülteni 2013;47(2):74-78
'Konya Eğitim ve Araştırma Hastanesi, Genel Cerrahi Kliniği, Konya-Türkiye

Yazışma Adresi / Address reprint requests to: Kemal Arslan, Konya Eğitim ve Araştırma Hastanesi Genel Cerrahi Kliniği, Hacı Şaban Mah. Meram Yeniyol Caddesi No:97 PK: 42090 Meram, Konya-Türkiye

Telefon / Phone: +90-332-444-0642

E-posta / E-mail: arslanka74@hotmail.com

Geliş tarihi / Date of receipt: 10 Kasım 2012 / November 10, 2012

Kabul tarihi / Date of acceptance: 17 Mayıs 2013 / May 17, 2013 


\section{GíRiş}

Tiroid ameliyatları tüm dünyada endokrin ve cerrahi kliniklerinde ensık uygulanan cerrahi girişimdir. En sık tiroidektomi endikasyonu malignite ve malignite şüphesi, kozmetik nedenler, bası belirtileri ve hipertiroidizmdir.

Tiroidektomimortalitesi ve morbiditesi gelişen cerrahi teknikler sayesinde oldukça azalmış olmasına rağmen hala devam etmektedir. Morbidite oranı $\% 13$ ve ciddi komplikasyon \%2 civarındadır (1). Yakınzamanlara kadar cerrahi gerektiren selimtiroid hastalıklarının tedavisinde bilateral subtotal tiroidektomi (BST) uygulanmaktaydı $(2,3)$. Son yıllarda maligntiroid hastalıklarının yanında selim tiroid hastalıklarında da total tiroidektomi(TT) uygulanmakta ve önerilmektedir (4). Ancak TT'nin komplikasyonlarının BST'den daha fazla olduğu geçmişte bildirilmiş ve bu endişe halen devam etmektedir (5-7). Bizde kliniğimizde 2006 yılından itibaren selim tiroid hastalıklarının tedavisinde aşamalı olarak BST'den TT'ye geçmiş bulunmaktayız. Bu çalışmada amacımız selim tiroid hastalıklarının tedavisinde ST ile TT ameliyatlarının erken ve geç dönem komplikasyonlarının karşılaştırılmasıdır.

\section{HASTALAR VE YÖNTEM}

Konya Eğitim ve Araştırma Hastanesi Genel Cerrahi Kliniğinde 2004-2007 yılları arasında cerrahi gerektiren multinodüler guatr nedeniyle yapılan BST ile 2007-2008 yılları arasında yapılan TT ameliyatlarının erken ve geç dönem komplikasyonları hasta dosyalarından ve bilgisayar kayıtlarından retrospektif olarak incelendi. Çalışmaya dahil edilme kriteri preoperatif olarak multinodüler guatr tanısı olması ve postoperatif olarak bu tanının patolojik olarak teyid edilmesiydi. BST ve TT ameliyatı yapılan hastalar çalışmaya alındı. Malign ve nüks olgular, hipertiroidik vakalar, tek taraflı yapılan tiroidektomiler, daha önce boyun bölgesine radyoterapi anamnezi olan hastalar, ameliyat öncesi vokal kord paralizisi olanlar çalışma dışı bırakıldı. Hastaların demografik verileri, yapılan ameliyat, erken postoperatif ve geç dönem komplikasyonlar, takip süreleri kaydedildi. Hastalara preoperatif dönemde ve postoperatif 1 . günde Kulak
Burun Boğaz kliniğinde indirekt laringoskopi ile vokal kordları değerlendirildi. Vokal kord paralizisi gelişen hastalarda ameliyat sonrası 6. aya kadar düzelen hastalar geçici rekürren laringeal sinir paralizisi, 6. aydan sonra halen devam eden hastalar kalıCı paralizi olarak kabul edildi. Kalsiyum seviyeleri tüm hastalarda ameliyat sonrası takip edildi, $8 \mathrm{mg} /$ dl'den düşük olanlar, semptom ve muayene bulgusu olan hastalar hipokalsemi olarak kabul edildi. Ameliyat sonrası gelişen ve ilk 6 ayda düzelen hipokalsemiler geçici, 6.aydan sonra devam edenler kalıcı hipokalsemi olarak değerlendirildi.

\section{İstatistiksel Analiz}

İstatistik analizler SPSS 10 (SPSS, Inc., Chicago, IL, ABD) programında yapıldı. Veriler ortalama ve Standart Sapma olarak sunuldu. Gruplar arasında parametrik verilerin karşılaştırılmasında $T$ testi, nonparametrik verilerin karşılaştırılmasında ki-kare testi uygulandı. $\mathrm{P}<0.05$ değerleri istatistiksel olarak anlamlı kabul edildi.

\section{BULGULAR}

Çalışma süresince yaşları 18 ile 78 arasında değişen (ortalama 47.27 \pm 15.65 ) toplam 245 hastaya tiroidektomi ameliyatı yapıldı. Ameliyat sonrası patoloji raporlarında malign hastalık tespit edilen 12 hasta ve tek taraflı girişim yapılan 5 hasta çalışma dışı bırakıldı. Toplam 22 hasta takiplerine gelmediğinden ve ulaşılamadığından çalışmadan çıkarıldı. Çalışma akışı Şekil 1'de gösterilmiştir. Böylece BST grubunda 96, TT grubunda 110 hastanın analizi yapıldı. Çalışmaya

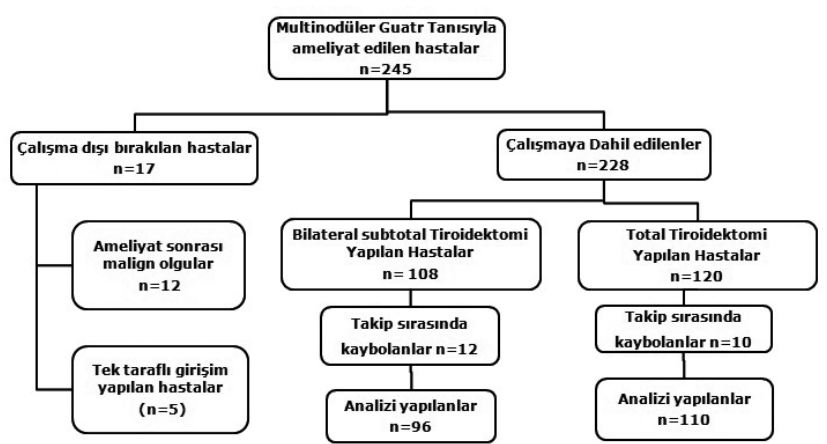

Şekil 1: Multinodüler guatr tanılı hastalarda çalışma akış şeması 
Tablo 1: Hastaların tedavi gruplarına göre özellikleri ve erken komplikasyonları

\begin{tabular}{lccc}
\hline & Subtotal Tiroidektomi $\mathbf{n = 9 6}$ & Total Tiroidektomi $\mathbf{n = 1 1 0}$ & P değeri \\
\hline Cinsiyet & & & \\
Kadın n(\%) & $81(84.4)$ & $94(85.5)$ & $16(14.5)$ \\
Erkek n(\%) & $15(15.6)$ & $45.7 \pm 15.6$ & 0.490 \\
Yaş, yıl (median) & $49.1 \pm 15.6$ & $52.7 \pm 5.3$ & 0,124 \\
Takip süresi, ay (median) & $69.4 \pm 8.3$ & & 0.001 \\
Erken Komplikasyonlar n(\%) & & $100(90.5)$ & $1(0.9)$ \\
Dren gereksinimi & $94(97.9)$ & $4(4.5)$ & 0.029 \\
Erken kanama nedeni ile reoperasyon & $1(1.0)$ & $1(1.0)$ & 0.716 \\
Flep ödemi & $5(5.2)$ & $5(4.5)$ & 0.415 \\
Hematom & $4(4.2)$ & $2(1.8)$ & 0.145 \\
Seroma & $3(3.1)$ & $10(9.1)$ & 0.438 \\
Enfeksiyon & $1(1.0)$ & $7(6.4)$ & 0.551 \\
Geçici hipokalsemi & $7(7.3)$ & & 0.417 \\
RLN geçici paralizisi & $5(5.2)$ & $3(2.7)$ & 0.481 \\
Geç Komplikasyonlar & & $2(1.8)$ & 0.564 \\
Kalıcı Hipokalsemi & $2(2.1)$ & $0(0)$ & 0.551 \\
RLN kalıcı paralizisi & $1(1.0)$ & 6.009 \\
Nüks & $6(6.3)$ & & \\
\hline
\end{tabular}

RLN: Rekürren laringeal sinirin

alınan hastalarda mortalite gözlenmedi.

Gruplardaki hasta sayıları, demografik özellikleri ve gelişen komplikasyonlar Tablo 1'de ayrıntılı olarak gösterilmiştir. Hastaların $175^{\prime} \mathrm{i}$ erkek ve $31^{\prime} \mathrm{i}$ kadındı. Gruplar arasında cinsiyet açısından istatistiksel olarak anlamlı fark yoktu. Hastaların yaş ortalaması BST grubunda 49.1 \pm 15.6 , TT grubunda $45.7 \pm 15.6$ idi ve gruplar açısından istatistiksel olarak anlamlı fark yoktu.

Takip süresi BST grubunda $69.4 \pm 8.3$ ay $(60-84)$, TT grubunda $52.7 \pm 5.3$ ay (48-60) idi. Ameliyat esnasında BST grubunda dren gereksinimi istatiksel anlamlı olarak daha fazla $(n=94, \% 97.9)$ idi. Erken postoperatif dönemde BST grubunda bir hastanın ameliyatının bitmesinden hemen sonra hızla büyüyen hematom nedeniyle tekrar ameliyat edildi ve üst pol bölgesinden superior tiroid arter lojundan kanama olduğu tespit edilerek hemostaz yapıldı. TT grubunda da bir hastada erken postoperatif 8 . saatte ameliyat bölgesinde gelişen hematom ve buna bağlı asfiksi nedeniyle, hastanın hematomu hasta yatağında boşaltılarak ameliyata alındı, bu hastada inferior tiroid arter lojundan kanama olduğu saptandı ve hemostaz yapıldı.

En sık tespit edilen komplikasyon her iki grupta toplam 17 hastada (\%8.3) geçici hipokalsemi idi. BST grubunda 10 hastada (\%9.1), TT grubunda 7 hastada (\%7.3) saptandı, gruplar arasında istatistiksel olarak anlamlı fark yoktu.

Geçici rekürren laringeal paralizisi toplam 12 hastada (\%7.2) geliş̧i. BST grubunda 5 hastada (5.2), TT grubunda 7 hastada (\%6.4) saptandı, gruplar arasında istatistiksel olarak anlamlı fark yoktu.

Hematom 5 (\%2.4), seroma 8 (\%3.9), flep ödemi 9 (\%4.4) hastada gelişti. Bu komplikasyonlar açısından gruplar arasında anlamlı bir fark yoktu. Hipokalsemi gelişen toplam 22 hastanın 17'si 6.ay içinde (\%77.3) düzeldi, sonuç olarak kalıcı hipokalsemi BST grubunda 2 hastada (\%2.1), TT grubunda 3 hastada (\%2.7) gelişti ve iki grup arasında istatistiksel olarak anlamlı fark yoktu.

Rekürren laringeal paralizisi gelişen 15 hastanın 12 'sinde (\%80) 6. ayın sonunda paralizi düzeldi ve toplam 3 hastada (\%1.5) paralizi ısrar etti ve kalıCı paralizi olarak kabul edildi. Kalıcı rekürren laringeal paralizisi açısından gruplar arasında istatistiksel olarak anlamlı fark yoktu.

BST grubunda $6(\% 6.3)$ hastada cerrahi girişim gerektiren nüks görüldü, TT grubunda görülmedi. Nüks açsından gruplar arasında anlamlı fark vardı $(p<0.05)$. Nüks görülen hastalardan 2'sine TT yapıldı ve herhangi bir komplikasyon görülmedi. 


\section{TARTIŞMA}

Multinodüler guatr ve Graves hastalığı gibi selim tiroid hastalıklarında BST'den, totale yakın ve TT'ye kadar uzanan bir yelpazede tedavi seçenekleri tartışılmaktadır. Çalışmamızda multinodüler guatr nedeniyle BST ve TT yapılan hastaların erken geç dönem komplikasyonları karşılaştırıldı.

Son yıllarda BSTnin yerini TT almıştır. Ancak $\mathrm{TT}^{\prime}$ de BST'ye göre komplikasyonların daha fazla olacağı endişesi halen devam etmektedir (8). Multinodüler guatr tanısıyla ameliyatı planlanan hastalarda en uygun teknik ve cerrahi yöntemin nasıl olması gerektiği literatürde uzun süredir tartışılan bir konudur (9). Komplikasyon oranının daha düşük olması ve ilaçsız ötiroid durumu sürdürme beklentisi multinodüler guatrın tedavisinde standart tedavi olarak düşünülmüştür (6).

BST'nin en önemli sorunlardan biri çıkarılan dokuda saptanan insidental tiroid malign hastalıktır ve bu durumda tamamlayıc tiroid ameliyatını gerekmesidir $(10,11)$. İnsidental tiroid malign hastalık nedeniyle uygulanan tamamlayıcı tiroidektominin komplikasyon riski primer ameliyatlardan daha yüksektir (12). Bizim daha önce yaptığımız bir çalışmada benign hastalık nedeniyle ameliyat edilen 505 tiroidektomi piyesinde \%10.9 oranında insidental malign tiroid hastalığını tespit ettik (13). Literatürde multinodüler guatr nedeniyle daha önce ameliyat edilen hastaların bakiye dokularında \%10-22'sinde insidental tiroid malign hastalığı saptanmıştır $(14,15)$.

Aslında temel sorun BST sonrası nükstür. Bilateral multinodüler guatr tüm bezi yaygın olarak tutmakta ve tedavisinde BST yapıldı ̌̆ında anormal doku geride kalabilmektedir. Uzun sürede de bu dokudan nüks gelişebilmektedir. Literatürde takip süreleri farklı olmasına rağmen BST sonrası nüks $\% 7.1$ ile $\% 70$ oranında bildirilmektedir (16-18). Bizim çalışmamızda 69 ay takip süresinde BST yapılan hastaların \%6.3'ünde nüks gelişmiştir. Daha uzun süreli takip süresinde de nüks gelişme ihtimali mevcuttur. TT grubunda nüks gelişmemiştir.

Nüks multinodüler guatr nedeni ile uygulananan tiroidektomide primer TT'ye göre kalıcı komplikasyon riski 5 kat artmaktadır (18-20). Rios ve arkadaşları yaptıkları çalışmalarında multinodüler guatrda
BST sonrası nüks oranının 5.yılda \%20, 15.yılda \%50 olduğunu bildirmişlerdir (18). Erbil ve arkadaşları (21) nüks oranının bırakılan fazla miktardaki doku ile direkt ilgili olduğunu bildirmişlerdir.

TT'nin BST'ye göre en önemli avantajı nüks olasılığını ve insidental tiroid malign hastalığı için gerekli olan tamamlayıcı tiroidektomi olasılığını ortadan kaldırmasıdır. Ancak TT'nin yüksek komplikasyon riski endişesi mevcuttur. Tiroidektomi sonrası en önemli komplikasyonlar hipoparatiroidi ve rekürren sinirin paralizisidir. Thomusch ve arkadaşları çok merkezli çalışmalarında tiroidektomi genişliğini komplikasyon riskini arttıran bir faktör olduğunu bildirmişlerdir (6). Günümüzde uygun cerrahi teknik ve artan deneyim ile TT'nin kalıcı komplikasyon oranını arttırmadan uygulanabileceğini gösteren bir çok çalışma vardır $(4,7,23)$.

Tiroid cerrahisinden sonra hipokalseminin nedeni paratiroid bezlerinin beslenmesinin bozulması, cerrahi olarak çıkarılması veya elektrokoterle bezlerin hasarlanmasına bağlı olabilir. Geçici hipokalsemi çok nadir bir durum değildir ve \%1 ile \% 40 oranında bildirilmiştir $(17,22-24)$. Bizim çalışmamızda da BST grubunda \%7.3 ve TT grubunda \%9.1 oranında saptanmıştı, gruplar arasında istatistiksel olarak anlamlı fark yoktu.

Kalıcı hipokalsemi BST sonrası \%0.8-1.9, TT sonrası \%0.4-13.8 oranında bidirilmiştir $(17,22-24)$. Bizim çalışmamızda BST sonrası \%2.1 ve TT sonrası $\% 2.8$ oranında kalıcı hipokalsemi saptadık ve yine her iki grup arasında istatistiksel olarak anlamlı fark yoktu.

Tiroidektominin en korkulan komplikasyon rekürren laringeal sinir paralizisidir. Geçici veya kalıcı ses kısıklığına yol açabilen bu durum hastanın sosyal yaşantısını oldukça etkileyen, istenmeyen bir durumdur. Literatürde geçici vokal kord paralizisinin \%0-10 arasında olduğu bildirilmiştir $(12,24)$. Bizim çalışmamızda geçici vokal kord paralizisi $\mathrm{BST}^{\prime}$ de $\% 5.2, \mathrm{TT}^{\prime}$ de $\% 6.4$ oranında görülmüştü. Biz bunun nedeninin ödem ve bazen sinirin tüm trase boyunca takip edilememesi, bu alanda hemostaz için kullanılan sütürlerle sinirin gerilmesi olduğunu düşünüyoruz.

Kalıcı rekürren laringeal sinir paralizisi yapılan çalışmalarda \%0-5 oranında bildirilmektedir $(12,22-$ 
24). Erbil ve arkadaşları da 3250 tiroidektomi vakasında kalıcı rekürren sinir paralizisi oranını \%1.8 olarak bildirmiş̧lerdir (21). Bizim çalışmamızda kalıcı rekürren sinir paralizisi BST grubunda \%1.0 ve TT grubunda \%1.8 oranındaydı ve gruplar arasında istatistiksel olarak anlamlı fark yoktu.

Bizim bu çalışmamızda BST ile TT ameliyatları sonrası erken ve geç komplikasyonların insidansında istatistiksel olarak anlamlı derecede fark olmamıştır.

\section{KAYNAKLAR}

1. Sadler GP, Clark OH, Van Heerden JA, Farley DR. Thyroid and parathyroid. In: Schwartz SI (Ed.). Principles of Surgery. $7^{\text {th }}$ ed. New York: McGrawHill Co; 1999. p.1661-713.

2. İ̧gör A(Editör). Tiroidektomi. In: Tiroit Hastalıkları ve Cerrahisi. 1.baskı, Istanbul Avrupa Tıp Kitapçılık Ltd Şti. 2000: 551-82.

3. işgör A (Editör). Hipertiroidizmin cerrahi tedavisi. In Tiroit Hastalıkları ve Cerrahisi.1.baskı, Istanbul Avrupa Tıp Kitapçılık Ltd Şti.2000: 331-43.

4. Agarwal G, Aggarwal V. Is total thyroidectomy the surgical procedure of choice for benign multi-noduler goiter? An evidence-based review. World J Surg 2008; 32(7): 1313-24.

5. Bozdağ AD, Çevikel MH, Demirkıran AE, Erpek H, Boylu Ş, Özgün H. Benign Tiroid Cerrahisinde Postoperatif Komplikasyonları Etkileyen Risk Faktörleri. ADÜ Tıp Fakültesi Dergisi 2002; 3(3): 25-9.

6. Thomusch $O$, Sekulla $C$, Dralle $H$. Is primary total thyroidectomy justified in benign multinodular goiter? Results of a prospective quality assurance study of 45 hospitals offering different levels of care. Chirurg. 2003; 74(5):437-43.

7. Erbil Y, Barbaros U, Salmaslioğlu A, Yanik BT, Bozbora A, Ozarmağan $S$. The advantage of near-total thyroidectomy to avoid postoperative hypoparathyroidism in benign multinodular goiter. Langenbecks Arch Surg. 2006; 391(6): 567-73.

8. Sayek $i$ (Editör). Tiroidektomi ve komplikasyonları. In: Temel Cerrahi 3. baskı; Ankara, Güneş Kitabevi Ltd. Şti.2004:1621-30.

9. Zambudio AR, Rodríguez J, Riquelme J, Soria T, Canteras M, Parrilla P. Prospective study of postoperative complications after total thyroidectomy for multinodular goiters by surgeons with experience in endocrine surgery. Ann Surg. 2004; 240(1): 18-25.

10. Tezelman S, Borucu I, Senyurek Giles Y, Tunca F, TerziogluT. The change in surgical practice from subtotal to near-total or total thyroidectomy in the treatment of patients with benign multinodular goiter. World J Surg. 2009; 33(3):400-5.

11. Giles Y, Boztepe H, Terziog/u T, Tezelman S. The advantage of total thyroidectomy to avoid reoperation for incidental thyroid cancer in multinodular goiter. Arch Surg 2004; 139(2):179-82.

\section{SONUÇ}

Cerrahi gerektiren selim tiroid hastalıklarında TT erken ve geç komplikasyonları arttırmamaktadır. BST sonrası nüks ihtimali vardır ve tekrar ameliyat gerektirebilmektedir. Nüks tiroid ameliyatlarında primer ameliyatlara göre komplikasyonlar belirgin şekilde daha fazladır. Bu nedenle benign tiroid hastalıklarında eğer cerrahi gerekliyse TT uygulanmalıdır.

12. Pezzullo L, Delrio P, Losito NS, Caracò C, Mozzillo N. Postoperative complications after completion thyroidectomy for differentiated thyroid cancer. Eur J SurgOncol. 1997; 23(3):215-8.

13. Arslan K, Eryılmaz MA, Eroğlu C, Karahan Ö. Total tiroidektomi uygulanan benign tiroid hastalıklı olgularda rastlantısal tiroid kanseri riski. Genel Tip Derg 2010;20(1):19-22.

14. Levin KE, Clark AH, Duh QY, Demeure M, Siperstein AE, Clark $\mathrm{OH}$. Reoperative thyroid surgery. Surgery 1992; 111: 604-9.

15. Menegaux F, Turpin G, Dahman $M$, et al. Secondary thyroidectomy in patients with prior thyroid surgery for benign disease: A study of 203 cases. Surgery 1999; 126(3): 479-83.

16. Lefevre JH, Tresallet $C$, Leenhardt L, Jublanc C, Chigot JP, Menegaux F. Reoperative surgery for thyroid disease. Langenbecks Arch Surg 2007; 392(6): 685-691.

17. Ozbas S, Kocak S, Aydintug S, Cakmak A, Demirkiran MA, Wishart GC. Comparison of the complications of subtotal, near total and total thyroidectomy in the surgical management of multinodular goitre. Endocr J 2005; 52(2): 199-205.

18. Ríos A, Rodríguez JM, Galindo PJ, Montoya MJ, Canteras M, Parrilla P. Surgical treatment of multinodular goiter in young patients. Endocrine 2005; 27(3): 245-52.

19. Röjdmark J, Järhult J. High long term recurrence rate after subtotal thyroidectomy for nodular goitre. Eur J Surg 1995; 161(10): 725-7.

20. Reeve TS, Delbridge L, Brady P, Crummer P, Smyth C. Secondary thyroidectomy: A twenty-year experience. World J Surg 1988; 12(4): 449-53.

21. Erbil $Y$, Barbaros $U$, Issever $H$, et al. Predictive factors for recurrent laryngeal nevre palsy and hypoparathyroidism after thyroid surgery. Clin Otolaryngol 2007;32(1):32-7.

22. Delbridge L, Guinea Al, Reeve TS. Total thyroidectomy for bilateral benign multinodular goiter: Effect of changing practice. ArchSurg 1999;134(12):1389-93.

23. Koyuncu A, Dökmetas HS, Turan M, et al. Comparison of different thyroidectomy techniques for benign thyroid disease. Endocr J 2003;50(6):723-7.

24. Jamski J, Jamska A, Graca M, Barczyński M, Włodyka J. Recurrent laryngeal nerve injury following thyroid surgery. Przegl Lek. 2004;61(1):13-6. 